\title{
1 Temperature regulation as a tool to program synthetic microbial community composition
}

2 Authors: Adam Krieger ${ }^{1}$, Jiahao Zhang ${ }^{2}$, Xiaoxia Nina Lin ${ }^{2,3}$

$3{ }^{1}$ Cellular and Molecular Biology Program, University of Michigan, Ann Arbor, MI 48103, USA

$4 \quad{ }^{2}$ Department of Chemical Engineering, University of Michigan, Ann Arbor, MI 48103, USA

$5{ }^{3}$ Department of Biomedical Engineering, University of Michigan, Ann Arbor, MI 48103, USA

6 Corresponding author: Xiaoxia Nina Lin, 2800 Plymouth Road, Ann Arbor, MI 48109-2800, B28-G054W

$7 \quad$ NCRC, (734) 647-8026, ninalin@umich.edu

8 Grant Numbers: AGK was supported by NIH T-32-GM007315 


\section{Abstract}

Engineering of synthetic microbial communities is emerging as a powerful new paradigm for performing

various industrially, medically, and environmentally important processes. To reach the fullest potential, however, this approach requires further development in many aspects, a key one being regulating the community composition. Here we leverage well established mechanisms in ecology which govern the relative abundance of multi-species ecosystems and develop a new tool for programming the composition of synthetic microbial communities. Using a simple model system consisting of two microorganisms Escherichia coli and Pseudomonas putida, which occupy different but partially overlapping thermal niches, we demonstrate that temperature regulation can be used to enable coexistence and program the community composition. We first investigate a constant temperature regime and show that different temperatures lead to different community compositions. Next, we invent a new cycling temperature regime and show that it can dynamically tune the microbial community, achieving a wide range of compositions depending on parameters that are readily manipulatable. Our work provides conclusive proof of concept that temperature regulation is a versatile and powerful tool capable of programming compositions of synthetic microbial communities. Keywords: synthetic microbial communities, ecology, temperature, community composition

\section{Introduction}

Microbial systems have been used for decades to perform functions with industrial, environmental, and medical relevance (Bouwer \& Zehnder, 1993; Göddel et al., 1979; Houde, Kademi, \& Leblanc, 2004). Traditionally, a single microbial species is cultured for a specific functionality. If necessary, genetic modifications are made to this organism to enable novel capabilities. As the field surrounding this paradigm has progressed, however, challenges faced by this monoculture approach have become 
as metabolic burden, a lack of or limited intracellular compartmentalization, and energetic tradeoffs

34 (Litchman, Edwards, \& Klausmeier, 2015; Zhou, Qiao, Edgar, \& Stephanopoulos, 2015). At the population level, monocultures are susceptible to invasion and subsequent population collapse by agents such as foreign bacteria, fungi, and bacteriophages. One approach to address these challenges is utilization of synthetic microbial communities as opposed to monocultures for biotechnology applications. Microbial communities enable division of labor and specialization within a population, decreasing metabolic burden and enabling reaction compartmentalization. More diverse microbial communities can also exhibit increased stability (Yurtsev, Conwill, \& Gore, 2016) and resistance to invasion by foreign agents (Dirk et al., 2012), as well as increased biomass production (McGrady-Steed, Harris, \& Morin, 1997) and nutrient utilization (Ptacnik et al., 2008) in certain contexts.

While the potential advantages offered by a microbial community over a monoculture are clear, community based approaches face challenges of their own. One of the most basic challenges is control of community composition. Community composition can have different meanings in different ecological contexts, but here is used to refer specifically to the relative abundance of individual species within the community. In a microbial community in which each species exhibits a specific and unique function within the community, the metabolic functionality of the community as a whole can depend greatly on the relative abundance of each species within the community (Hsu et al., 2019; Scholz, Graves, Minty, \& Lin, 2017; H. Zhang, Pereira, Li, \& Stephanopoulos, 2015). Despite significant advances in many related research topics, progress in control of synthetic microbial community compositions has been slow, likely

52 due at least in part to a dearth of tools capable of dynamically modulating relative species abundance.

53 The most commonly used technique is manipulation of inoculation ratio (Minty et al., 2013; Shong,

54 Rafael, Diaz, \& Collins, n.d.; Strickland, Lauber, Fierer, \& Bradford, 2009), but this approach is not 
interactions between community residents the community composition often quickly shifts away from

57 this initial condition.

While synthetic biology researchers are starting to develop new approaches to address the aforementioned challenges, mechanisms that enable species coexistence and modulate relative abundance over time in complex naturally occurring communities have been a major theme in

61 ecological study for almost 100 years. The current state of the field proposes that all mechanisms that

62 maintain and modulate species diversity operate through one of two broad classes, equalizing

63 mechanisms and stabilizing mechanisms (Chesson, 2000). Equalizing mechanisms lessen the relative

64 fitness differences between species (which can be more simply conceptualized as growth rates, although

65 in truth this is a slight oversimplification), and stabilizing mechanisms increase the niche differences

66 between species, which is to say they decrease the extent to which species compete directly with each

67 other (Chesson, 2000). According to Chesson's theory, equalizing mechanisms can support coexistence by decreasing the difference between growth rates, but stabilizing mechanisms are required for

69 coexistence. This makes intuitive sense in that, if there is a difference between the growth rates of two species, no matter how small, eventually that difference will lead to competitive exclusion of the slower

71 growing species in the absence of additional compensatory mechanisms. Therefore, any environmental

72 variables which differentially affect growth rates or niche partitioning would be expected to influence

73 the ability of species to coexistence and/or community composition.

75 maintenance of species coexistence to a synthetic microbial community in order to develop tools that are capable of dynamically modulating relative species abundance. The modality we have implemented

77 is temperature regulation. Using a synthetic community consisting of two model microorganisms

78 Escherichia coli and Pseudomonas putida, which occupy different but partially overlapping thermal

79 niches, we demonstrate that temperature regulation is a tool that can be used to enable coexistence 
and program the community composition. Specifically, we develop two temperature mediated regimes.

81 First, using a constant temperature for the co-culture, we are able to identify a temperature window

82 that allows co-existence of the two microbes, albeit exhibiting only a small range of achievable

83 compositions. Next, we develop a novel regime of cycling between a high temperature favoring E. coli

84 and a low one favoring P. putida, which enables co-existence of the two microorganisms in a highly

85 tunable manner.

\section{Materials and Methods:}

\subsection{Microbial strains and cultivation}

E. coli $K 12$ substr. MG1655 constitutively expressing a chromosomally integrated YFP construct was generated by P1 transduction as described by Thomason et al.(Thomason, Costantino, \& Court, 2007)

92 cassette. The $P$. putida KT2440 strain constitutively expressing mCherry was a generous gift from Dr.

93 Esteban Martinez-Garcia of the Victor de Lorenzo lab. Addition of an mCherry cassette to P. putida

94 KT2440 was performed via Tn7 transposon assisted cloning (Koch, Jensen, \& Nybroe, 2001; Martínez-

95 García, Calles, Arévalo-Rodríguez, \& De Lorenzo, 2011).

$97 \mathrm{~g} / \mathrm{L} \mathrm{Na}_{2} \mathrm{HPO}_{4}$ anhydrous, $15 \mathrm{~g} / \mathrm{L} \mathrm{KH}_{2} \mathrm{PO}_{4}, 2.5 \mathrm{~g} / \mathrm{L} \mathrm{NaCl}, 5 \mathrm{~g} / \mathrm{L} \mathrm{NH}_{4} \mathrm{Cl}$ ], $20 \mathrm{~mL} 20 \%$ glucose, $2 \mathrm{~mL} 1 \mathrm{M} \mathrm{MgSO}$,

$98100 \mathrm{uL} 1 \mathrm{M} \mathrm{CaCl}_{2}, 780 \mathrm{~mL}$ sterile deionized $\mathrm{H}_{2} \mathrm{O}$; autoclave all components separately then mix under 99 sterile conditions). 
$10150 \mathrm{uL}$ of log phase bi-culture was pipetted onto a slide and a coverslip was placed on top. Confocal

102 fluorescence microscopy was performed using an upright Olympus FV1200 confocal microscope

103 equipped with a $60 x$ objective and $405,488,515,543$, and 635 lasers. Images were exported and

104 analyzed with ImageJ.

2.3 Quantification of maximum specific growth rate in co-cultures

E. coli $K 12$ substr. MG1655-YFP and P. putida KT2440-mCh were seeded from $-80{ }^{\circ} \mathrm{C}$ cryostocks and grown in monoculture overnight to stationary phase in $14 \mathrm{~mL}$ Corning Falcon ${ }^{\circledR}$ test tubes (polypropylene test tube, round bottom, 17x100mm, 14mL, graduated, with clear snap cap, Sterile, 25 per Pack) in a 2 $\mathrm{mL}$ volume of M9 minimal media. Overnight cultures were diluted 1:100 into fresh M9 media and grown into exponential growth phase $\left(\mathrm{OD}_{600} \sim 0.4-0.6\right)$. The cell density of the two cultures of exponential phase cells were normalized to each other using $\mathrm{OD}_{600}$ and then diluted 1:100 into fresh $\mathrm{M} 9$ media in a Greiner Bio-one CELLSTAR ${ }^{\text {TM }} 96$ well $\mu$ Clear flat bottomed microplate with a $200 \mathrm{uL}$ final volume. Strains were grown in monoculture and co-culture in triplicate $(2 \mathrm{uL}$ of the desired strain was added for monocultures, and 1 uL each of both strains was added for co-cultures to 198 uL M9 media).

115 These nine wells were surrounded by wells filled with $200 \mathrm{uL}$ sterile deionized $\mathrm{H}_{2} \mathrm{O}$ to inhibit evaporation

116 from experimental wells. The lid was treated with a mixture of $20 \%$ ethanol $+0.5 \%$ Triton X-100 to

117 avoid condensation formation on the lid (pour enough mixture to completely cover the bottom of the

118 lid, let sit 5 minutes, pour off and let air dry). The lid was taped on using Fisherbrand ${ }^{\mathrm{TM}}$ labeling tape.

119 The plate was incubated in a Biotek Synergy H1 platereader for 24 hours with plate reads every 10

120 minutes and continuous orbital shaking at $282 \mathrm{cpm}$. At each timepoint, reads at $600 \mathrm{~nm}$ wavelength,

121 Excitation: $510 \mathrm{~nm}$ Emission: $540 \mathrm{~nm}$, and Excitation: $585 \mathrm{~nm}$ Emission: $620 \mathrm{~nm}$ were taken of each well

122 (these wavelengths were empirically determined to maximize the specific signal and minimize crosstalk

123 between the two channels for our constructs, media, and platereader; data not shown). The maximum 
124 specific growth rates from the co-culture growth curves were calculated by fitting the early exponential

125 growth phase to an exponential function using Microsoft Excel.

\subsection{Constant temperature regime: culture growth and community composition quantification}

127 E. coli K12 substr. MG1655-YFP and P. putida KT2440-mCh were seeded from $-80^{\circ} \mathrm{C}$ cryostocks and

128 grown in monoculture overnight to stationary phase in $14 \mathrm{~mL}$ Corning Falcon ${ }^{\circledR}$ test tubes (polypropylene

129 test tube, round bottom, 17x100mm, 14mL, graduated, with clear snap cap, Sterile, 25 per Pack) in a 2

$130 \mathrm{~mL}$ volume of M9 minimal media. Overnight cultures were diluted 1:100 into fresh M9 media and

131 grown into exponential growth phase (OD600 0.4-0.6). The cell density of the two cultures of

132 exponential phase cells were normalized to each other using $\mathrm{OD}_{600}$ and then diluted into $14 \mathrm{~mL}$ Corning

133 Falcon ${ }^{\circledast}$ test tubes with $2 \mathrm{~mL}$ fresh M9 at three different inoculation ratios; 1:10, 1:1, and 10:1 E. coli : P.

134 putida (2 uL E. coli +18 uL P. putida, $10 \mathrm{uL}$ E. coli $+10 \mathrm{uL}$ P. putida, and $18 \mathrm{uL}$ E. coli $+2 \mathrm{uL}$ P. putida).

135 Each inoculation ratio was performed in triplicate producing a total of nine cultures. Cultures were

136 incubated in a Lab-Line Instruments Model No. 3528 incubator with a New Brunswick Scientific C1

137 platform shaker placed inside for shaking set at a speed of 50 (no units are indicated on the shaker). The

138 temperature was monitored throughout the lifetime of each experiment using an Omega OM-91

139 portable temperature data logger (see Supplemental Information). Each culture was passaged 1:100

140 into $2 \mathrm{~mL}$ fresh M9 media in $14 \mathrm{~mL}$ Corning Falcon ${ }^{\circledR}$ test tubes twice daily (8 hour and 16 hour growth

141 periods). At each passaging time point, each culture was vortexed for 5 seconds and a $1 \mathrm{uL}$ sample was

142 taken for quantification of community composition via flow cytometry. Passaging was performed after

143 vortexing.

1442.5 Cycling temperature regime: culture growth and community composition quantification

145 E. coli K12 substr. MG1655-YFP and P. putida KT2440-mCh were seeded from $-80^{\circ} \mathrm{C}$ cryostocks and

146 grown in monoculture overnight to stationary phase in $14 \mathrm{~mL}$ Corning Falcon ${ }^{\circledR}$ test tubes (polypropylene 
147 test tube, round bottom, $17 \times 100 \mathrm{~mm}, 14 \mathrm{~mL}$, graduated, with clear snap cap, Sterile, 25 per Pack) in a 2

$148 \mathrm{~mL}$ volume of M9 minimal media. Overnight cultures were diluted 1:100 into fresh M9 media and

149 grown into exponential growth phase $\left(\mathrm{OD}_{600} \sim 0.4-0.6\right)$. The cell density of the two cultures of

150 exponential phase cells were normalized to each other using $\mathrm{OD}_{600}$ and then diluted into a $14 \mathrm{~mL}$

151 Corning Falcon ${ }^{\circledast}$ test tube with $2 \mathrm{~mL}$ fresh M9 at a 1:1 inoculation ratio (10 uL E. coli $+10 \mathrm{uL}$ P. putida).

152 For the long-time interval cycling temperature program experiments, the culture was incubated at $37^{\circ} \mathrm{C}$

153 for 16 hours in a New Brunswick Scientific Excella E24 Incubator Shaker with 225 rpm shaking, and at 31

$154{ }^{\circ} \mathrm{C}$ for 8 hours in a Lab-Line Instruments Model No. 3528 with 225 rpm shaking. For the short-time

155 interval cycling temperature experiments, a culture was prepared as above and incubated for

156 approximately 72 hours to allow adaptation to culture conditions. The culture was then incubated at 27

$157{ }^{\circ} \mathrm{C}$ until the community composition reached a 1:1 ratio, and then was incubated at the indicated

158 temperatures for the indicated times. After each growth period, cultures were vortexed for 5 seconds

159 and a 1 uL sample was taken for quantification of community composition by flow cytometry, and then

160 the culture was passaged 1:100 into $2 \mathrm{~mL}$ of fresh $\mathrm{M9}$ media in a new $14 \mathrm{~mL}$ Corning Falcon ${ }^{\circledR}$ test tube.

\subsection{Quantification of community composition via flow cytometry}

Samples taken from co-cultures were diluted to $10^{6}$ cell $/ \mathrm{mL}$ in $1 \times$ PBS $(137 \mathrm{mM} \mathrm{NaCl}, 2.7 \mathrm{mM} \mathrm{KCl}, 10$ $\mathrm{mM} \mathrm{Na}_{2} \mathrm{HPO}_{4}, 1.8 \mathrm{mM} \mathrm{KH}_{2} \mathrm{PO}_{4} ; \mathrm{pH} 7.4$ ) and run on an Applied Biosystems Attune acoustic focusing cytometer. $300 \mathrm{uL}$ of sample was acquired by the device for each run and 10,000 events were recorded. The instrument configuration was as follows: (Threshold (x1000): FSC: 10; SSC: 10; BL1: 10; BL2: 10; BL3: 


\subsection{Modeling of population dynamics under the cycling temperature regime}

172 For the cycling temperature regime, consider each cycle consists of a time interval $t^{H}$ at a high

173 temperature favoring $E$. coli and then another time interval $t^{L}$ at a low temperature favoring P. putida.

174 Two types of models were developed. In the first one, simple exponential growth is assumed.

$$
\frac{d E}{d t}=r_{E} E \quad(\text { Eq. 2.7.1) }
$$

$$
\frac{d P}{d t}=r_{P} P \quad(\text { Eq. 2.7.2) }
$$

where variables $E$ and $P$ are $E$. coli and $P$. putida cell densities respectively; $r_{E}$ and $r_{P}$, their specific

growth rates respectively, are model parameters. We further denote $r_{E}^{H}$ and $r_{P}^{H}$ as the specific grow

rates of $E$. coli and P. putida respectively at the high temperature; $r_{E}^{L}$ and $r_{P}^{L}$ the specific grow rates of $E$.

$\Delta r^{H} \equiv r_{E}^{H}-r_{P}^{H}, \Delta r^{L} \equiv r_{P}^{L}-r_{E}^{L}$

explicit functions of time:

$$
\begin{aligned}
& E(t)=\left\{\begin{array}{cc}
E_{0} \cdot e^{r_{E}^{H} \cdot t}, & 0 \leq t \leq t^{H} \\
E_{0} e^{r_{E}^{H} \cdot t^{H}} \cdot e^{r_{E}^{L}\left(t-t^{H}\right)}, & t^{H} \leq t \leq t^{H}+t^{L}
\end{array}\right. \\
& P(t)=\left\{\begin{array}{cc}
P_{0} \cdot e^{r_{P}^{H} \cdot t}, & 0 \leq t \leq t^{H} \\
P_{0} e^{r_{P}^{H} \cdot t^{H}} \cdot e^{r_{P}^{L}\left(t-t^{H}\right)}, & t^{H} \leq t \leq t^{H}+t^{L}
\end{array}\right.
\end{aligned}
$$

where $E_{0}$ and $P_{0}$ are the E. coli and $P$. putida cell densities respectively at the beginning of the cycle.

$$
\frac{E\left(t^{H}+t^{L}\right)}{P\left(t^{H}+t^{L}\right)}=\frac{E_{0} e^{r_{E}^{H} \cdot t^{H}} e^{r_{E}^{L} \cdot t^{L}}}{P_{0} e^{r_{P}^{H} \cdot t^{H}} e^{r_{P}^{L} \cdot t^{L}}}=\frac{E_{0}}{P_{0}} \cdot e^{\left(r_{E}^{H}-r_{P}^{H}\right) t^{H}-\left(r_{P}^{L}-r_{E}^{L}\right) t^{L}}=\frac{E_{0}}{P_{0}}
$$


It follows that:

$$
\left(r_{E}^{H}-r_{P}^{H}\right) t^{H}-\left(r_{P}^{L}-r_{E}^{L}\right) t^{L}=0
$$

191 Therefore, we have the relationship (Eq. 3.4.1):

$$
\frac{t^{H}}{t^{L}}=\frac{r_{P}^{L}-r_{E}^{L}}{r_{E}^{H}-r_{P}^{H}}=\frac{\Delta r^{L}}{\Delta r^{H}}
$$

$$
\left(\frac{E}{P}\right)_{\text {average }}=\frac{\int_{0}^{t^{H}}\left(\frac{E_{0} \cdot e^{r_{E}^{H} \cdot t}}{P_{0} \cdot e^{r_{P}^{H} \cdot t}}\right) d t+\int_{t^{H}}^{t^{H}+t^{L}}\left[\frac{\left.E_{0} e^{r_{E}^{H} \cdot t^{H}} \cdot e^{r_{E}^{L}\left(t-t^{H}\right.}\right)}{\left.P_{0} e^{r_{P}^{H} \cdot t^{H}} \cdot e^{r_{P}^{L}\left(t-t^{H}\right)}\right] d t}\right.}{t^{H}+t^{L}}
$$

Simplifications of this expression, including utilization of the relationship above between time intervals

$$
\left(\frac{E}{P}\right)_{\text {average }}=\frac{E_{0}}{P_{0}} \cdot\left[\frac{\Delta r^{L}\left(e^{\Delta r^{H} \cdot t^{H}}-1\right)+\Delta r^{H}\left(e^{\Delta r^{L} \cdot t^{L}}-1\right)}{\Delta r^{H} \cdot \Delta r^{L} \cdot\left(t^{H}+t^{L}\right)}\right]
$$

$$
\frac{d E}{d t}=r_{E} E\left(1-\frac{E}{K_{E}}\right) \quad(\text { Eq. 2.7.7) }
$$

$$
\frac{d P}{d t}=r_{P} P\left(1-\frac{P}{K_{P}}\right) \quad(\text { Eq. 2.7.8) }
$$

201 where $r_{E}$ and $r_{P}$ are the maximum specific growth rates of $E$. coli and $P$. putida respectively; $K_{E}$ and $K_{P}$

202 are the corresponding carrying capacities. A Mathlab script (see Supplemental information) was

203 developed to simulate repeated cycles of cell growth at a high temperature and then low temperature

204 using these two un-coupled ordinary differential equations. 


\section{Results}

\subsection{Overall approach}

Our objective is to develop new tools for regulating synthetic microbial community compositions, by leveraging temperature-dependent growths of distinct desired community members. phenotypes at various temperatures, we propose two temperature mediated regimes for achieving coexistence and regulating community compositions. In the first regime (Fig. 1A), a constant temperature

212 is used for growing the bi-culture. It is expected that if a low temperature is selected, the microbe

213 favored at the temperature (i.e. exhibiting a higher growth rate) would dominate; and vice versa at a

214 high temperature. We hypothesize, however, for a small range of intermediate temperatures, the two

215 microbes could co-exist. In the second regime (Fig. 1B), the bi-culture is cycled between a low

216 temperature and a high one, which favor each of the two microbes respectively, and co-existence of the

217 two microbes are maintained over an extended period of time. Operating parameters include the high

218 and low temperatures, and the time durations the bi-culture spends at each temperature. By

219 manipulating these parameters, one would be able to regulate the average community composition and 220 its variation.

\subsection{Design and implementation of a model microbial bi-culture}

222 To explore the temperature regulation regimes proposed above, we first established a model synthetic

223 microbial system consisting of two well-characterized bacterial species. One is the soil bacterium

224 Pseudomonas putida KT2440 (Belda et al., 2016). P. putida is found throughout the natural

225 environment, predominantly in soil and plant rhizospheres (Belda et al., 2016; Thomas, Koh, Bankowski,

226 \& Seto, 2013). This bacterium is a mesophilic organism, growing optimally at relatively moderate

227 temperatures (Munna, M. S., Zeba, Z., and Noor, 2015), and has an extremely versatile metabolism 
which has earned it a reputation as an excellent bioremediation agent (Belda et al., 2016). In this study

we use a strain of $P$. putida which has been tagged with a constitutively expressed chromosomal copy of

bacterial species in our system is E. coli K12 MG1655 (Blattner et al., 1997). One of the most commonly

used model organisms in microbiology, E. coli can be either commensal or pathogenic gut colonizers of

241 (Supplemental Fig. 2 A) and flow cytometry (Supplemental Fig. 2 B). We then quantified the growth

242 phenotype of each species in co-culture at various temperatures. Particularly, we determined the

243 maximum specific growth rates at different temperatures (Fig. 2, referred to as temperature profiles

244 hereafter), using growth curves derived from population level fluorescence from each species in bi-

245 cultures. The temperature profiles indicate that for each species there exists a temperature range in

246 which the species would be expected to have a growth advantage over the other (when the

247 temperature is less than $35.5^{\circ} \mathrm{C}, P$. putida $\mu_{\max }>E$. coli $\mu_{\max }$ and when the temperature is greater than

$248 \sim 35.5^{\circ} \mathrm{C}$ then P. putida $\mu_{\max }<$ E. coli $\left.\mu_{\max }\right)$. 

contrast, in the temperature window where composition changes are milder, it is indeed possible to

We first explore the constant temperature regime by investigating the community population dynamics over time at various selected temperatures. In this series of experiments, the temperature was maintained at a constant value $\left( \pm 0.2^{\circ} \mathrm{C}\right.$, Supplemental Fig. 3$)$ throughout the entire course of an experiment, and co-cultures were diluted 1:100 into fresh media twice daily $(9 \mathrm{~h}$ and $15 \mathrm{~h}$ growth periods). Each experiment was conducted for times ranging from 60 to 400 hours depending upon the observed dynamics. Community composition was quantified via flow cytometry with a measurement taken at each dilution time point. The dynamics of the bi-culture is shown in Fig. 3A for three representative temperatures, $35.5^{\circ} \mathrm{C}, 36.5^{\circ} \mathrm{C}$, and $39.5{ }^{\circ} \mathrm{C}$; the full set of data for 10 temperatures in the range of $32-39.5^{\circ} \mathrm{C}$ are provided in detail in Supplemental Fig. 1 and summarized in Fig. 3B. It was found that at $35.5^{\circ} \mathrm{C}$ and lower, the community was dominated by $P$. putida when the culture composition reached equilibrium (which is defined herein as changing no more than $\pm 15 \%$ over at least 3 consecutive time points), regardless of imposed initial ratios of the two bacteria (for example, see Fig. 3A top row). On the other hand, at temperatures above $\sim 39{ }^{\circ} \mathrm{C}$ the community was dominated by E. coli (for example, see Fig. $3 \mathrm{~A}$ bottom row). Interestingly, at temperatures between $35.5^{\circ} \mathrm{C}$ and $39{ }^{\circ} \mathrm{C}$, coexistence of the two bacteria was observed (for example, see Fig. 3A middle row). It is worth noting that the community composition at equilibrium of this bi-culture depends on the temperature in a highly nonlinear manner. Specifically, the equilibrium composition changed very gradually in the relatively wide temperature window of $35.7-39.1^{\circ} \mathrm{C}$; whereas this property changed drastically over a much narrower temperature window of $35.5-35.7^{\circ} \mathrm{C}$. These differences have direct implications for practical applications where a constant temperature is employed. Given the resolution of temperature control (e.g. $\pm 0.2^{\circ} \mathrm{C}$ in our laboratory set-up), it is practically impossible to achieve a specific desired community composition in the small temperature window where the composition is highly sensitive to fluctuations in temperature. In 
that the range of community compositions achievable in this temperature window $(\sim 70-95 \%$ E. coli at

$\left.275 \quad 36.1-39.1^{\circ} \mathrm{C}\right)$ is rather limited.

\subsection{Regulating community composition using a cycling temperature program}

We next explored the effect of temperature on community composition using what we refer to as a cycling temperature regime. In this approach, two distinct temperatures are selected so that the higher temperature favors the growth of one species and the lower one the other species. With these two temperatures selected, the bi-culture is incubated in repeated cycles, of which each starts with growth at the higher temperature for a specific time internal and then switches to growth at the lower temperature for another specific time interval. The key parameters we can manipulate are the two time intervals spent at the two temperatures respectively. When they are selected properly, this scheme enables the two species to coexist. Moreover, the composition of the bi-culture can be tuned as desired and it is determined by a combination of the initial composition and the two time intervals. This can be illustrated analytically if we use a simple mathematical model where each species grows exponentially, which is a reasonable assumption when bacterial cells grow at low densities (i.e. far away from the carrying capacities). Specifically, the two species can be stably maintained provided that the two time intervals are chosen such that the following relationship is satisfied (see Materials and Methods for details):

$$
\frac{t^{H}}{t^{L}}=\frac{\Delta r^{L}}{\Delta r^{H}}=\frac{r_{P}^{L}-r_{E}^{L}}{r_{E}^{H}-r_{P}^{H}}
$$

where $t^{H}$ and $t^{L}$ are the time intervals the culture spends at the high temperature and low temperature respectively in each cycle. $r_{P}^{L}$ and $r_{E}^{L}$ denote the specific grow rates of $P$. putida and $E$. coli respectively at the low temperature. Note that $r_{P}^{L}>r_{E}^{L}$ and the difference is defined as $\Delta r^{L} \equiv r_{P}^{L}-r_{E}^{L}$. Similarly, 
$296 r_{P}^{H}$ and $r_{E}^{H}$ denote the specific grow rates of $P$. putida and $E$. coli respectively at the high temperature.

$297 r_{P}^{H}<r_{E}^{H}$ and the difference is defined as $\Delta r^{H} \equiv r_{E}^{H}-r_{P}^{H}$. The above equation shows that only the ratio

298 of the two time intervals matters and it is equal to the inverse of the ratio of specific growth rate

299 differences between the two species at the corresponding temperatures. This is a specific quantitative

300 relationship that only holds for the exponential growth model. However, certain qualitative aspects of

301 this relationship are generalizable. In particular, it can be expected that the time interval at which there

302 is a smaller grow rate difference between the two species needs to be longer.

In addition to enabling co-existence, the cycling temperature regime also provides an effective

means for tuning the community composition, which is determined by the two time intervals and the as follows (see Materials and Methods for details):

$$
\left(\frac{E}{P}\right)_{\text {average }}=\left(\frac{E}{P}\right)_{\text {initial }} \cdot\left[\frac{\Delta r^{L}\left(e^{\Delta r^{H} \cdot t^{H}}-1\right)+\Delta r^{H}\left(e^{\Delta r^{L} \cdot t^{L}}-1\right)}{\Delta r^{H} \cdot \Delta r^{L} \cdot\left(t^{H}+t^{L}\right)}\right]
$$

310 where $\left(\frac{E}{P}\right)_{\text {initial }}$ and $\left(\frac{E}{P}\right)_{\text {average }}$ represent the ratio of E. coli cells to P. putida cells at the beginning and

311 averaged over the cycle respectively. A desired average ratio, therefore, can be achieved when one sets

312 the initial ratio and the two time intervals appropriately. In more general cases where the simple

313 exponential growth model does not apply, the above well-structured analytical relationship may not

314 exist. However, there will still be general trends. For instance, the shorter the two time intervals are,

315 the closer the average ratio is to the initial one. Such design principles can be explored systematically

316 through numerical simulation for specific systems. 
Guided by the mathematical modeling and analysis described above, we experimentally

318 implemented the cycling temperature regime on the E. coli and P. putida bi-culture model system. We

319 were able to demonstrate that the bi-culture could be maintained over an extensive period of time

320 when the parameter settings were chosen appropriately. A representative example is shown in Fig. 4,

321 where $37^{\circ} \mathrm{C}$ was chosen as the high temperature favoring E. coli and $31{ }^{\circ} \mathrm{C}$ as the low temperature

322 favoring $P$. putida. The bi-culture was started with an inoculation ratio of 1:1 and incubated in a cycling

323 temperature program of 16 hours at $37^{\circ} \mathrm{C}$ and 8 hours at $31^{\circ} \mathrm{C}$. It was maintained stably at an average

324 composition of $45 \%$ E. coli and 55\% P. putida with a standard deviation of $27 \%$ for a total of 4 cycles (96

325 hours).

As illustrated in Fig. 4, the community composition exhibits dynamic oscillations under the

327 cycling temperature regime. The amplitude of the oscillation, i.e. to what extent the composition

328 fluctuates over time, is an important criterion that deserves closer examination. We investigated this

329 first through computational simulation. Here, we assumed no direct interactions between the two

330 species and used two un-coupled logistic growth equations to model the bi-culture incubated in a

331 cycling temperature program and diluted at the end of each time interval (see Materials and Methods

332 for details). Our results showed that the amplitude of the oscillation was dependent on the duration of

333 the time intervals. Longer time intervals result in larger amplitude oscillations in community

334 composition (Fig. 5A) and shorter time intervals lead to more desirable, smaller amplitude oscillations

335 (Fig. 5B). We next validated our model predictions with wet lab experiments. Fig. 6 illustrated the

336 results of an experiment in which we set the temperatures to be $27^{\circ} \mathrm{C}$ and $39^{\circ} \mathrm{C}$, which favor $P$. putida

337 and E. coli respectively, and incubated the bi-culture with shorter time intervals compared to those in

338 Fig. 4. The bi-culture was maintained at an average composition of $51 \%$ E. coli and $49 \%$ P. putida with a

339 standard deviation of $11 \%$, which was substantially smaller than that in Fig. 4, 27\%. This approach of

340 employing shorter time intervals can, theoretically, reduce the amplitude of oscillation in community 
341 composition to any arbitrarily small value. In practice, however, various constraints would arise. In

342 particular, it takes cells a certain amount of time to physiologically respond to changes in temperature,

343 which sets a lower limit on the time interval parameter in this cycling temperature regime.

\section{Discussion and Conclusion}

346 Synthetic microbial communities are increasingly being used for a variety of industrial, medical, and

347 environmental applications such as production of commodity chemicals, proteins, and bioremediation

348 (Höffner \& Barton, 2014; S. Zhang, Merino, Okamoto, \& Gedalanga, 2018). Because microbial

349 community function depends in part on the community composition, it is of inherent interest to

350 synthetic ecology, an emerging area of synthetic biology, that environmental conditions have been

351 demonstrated to affect community composition in an ecological context. While the synthetic

352 biology/ecology literature has yet to treat the subject thoroughly, ecologists have been studying the

353 effects of environmental factors on community composition for decades. It has been proposed and

354 demonstrated that fluctuations in environmental conditions such as nutrient availability and

355 temperature can enable coexistence and influence compositions of various natural communities (Harder

356 \& Veldkamp, 1971; Hutchinson, 1961; Jiang \& Morin, 2007; Tilman, 1999). Interestingly, in the

357 biotechnology context, the effect of modulating a variety of environmental parameters was explored in

358 the 1970's and 1980's (Davison \& Stephanopoulos, 1986). These previous studies, however, focused on

359 the chemostat setting, which is not commonly used in biotechnological applications. It is known that an

360 abundance of cellular processes such as nutrient sensing, signal transduction, and macromolecular

361 synthesis are affected by the constantly changing environment within a batch culture (Ziv, Brandt, \&

362 Gresham, 2013). Therefore the population dynamics in a batch culture are expected to differ

363 significantly from those seen in a chemostat culture. Accordingly, here we show that in sequential batch 
cultures, the environmental condition of temperature can be used to modulate growth rates and rationally regulate community composition in synthetic microbial communities.

374 interactions play a role. It is possible that interactions commonly observed between species in microbial

377 and could have effects on community composition. It would be interesting for future work to investigate 378 the underlying mechanisms of this intriguing observation. optimizing functionality in a biotechnological context. We demonstrate here that temperature regulation is one modality that can be used to rationally regulate composition of synthetic microbial communities. However, a variety of other modalities such as $\mathrm{pH}$, salt concentration, etc. can

383 theoretically be implemented similarly, assuming that the basic requirements we propose for 384 temperature are met (namely that one range of conditions favors growth of one species, and a second range of conditions favors growth of the other species). The advantage of temperature over some of

386 these other modalities is that it can be dynamically changed without requiring media replacement, and 387 is readily implementable. Other modalities for regulating synthetic microbial community composition 
have been explored in the literature, most notably by Spencer Scott et al. (Scott et al., 2017). In this innovative work, the authors designed and implemented a self-limiting synthetic quorum sensing regulated lysis system to prevent competitive exclusion and enable coexistence between otherwise incompatible community members. One advantage of their approach is the potential for scalability in community complexity, as there are a variety of orthogonal, well-characterized quorum sensing systems that could be utilized with different species or groups of species in a community. Whereas, the alternative approach demonstrated in this work has the advantage that because response to temperature is one of the oldest, core physiological responses of microbes, although adaptation to temperature is expected to diminish its effectiveness at some point, the time scales at which temperature regulation is able to enable coexistence and regulate community composition are quite long (up to 400 hours with our experimental system). However, it would not be straightforward to extend the cycling temperature regime to more complex communities; development of significantly more complicated control schemes will be required. along with the associated design principles we have established can be applied to a wide variety of

405 synthetic co-cultures. New approaches for regulating synthetic microbial community composition will 406 continue to emerge and we envision that temperature based control schemes will contribute to a 407 powerful toolbox in the future consisting of various well-developed modalities for regulating synthetic 408 and natural microbial communities, from which researchers can choose based on the specific properties and performance requirements of their system. 
412 AGK was supported by the NIH Cellular and Molecular Biology Training Grant T-32-GM007315 and an

413 Integrated Training in Microbial Systems fellowship. The authors would like to thank Scott Scholz for

414 helpful discussions.

415

416

\section{References}

Belda, E., Heck, R. G. A. Van, Fraser, C., Klenk, H., Sekowska, A., Vallenet, D., \& Martins, V. A. P. (2016).

Blattner, F. R., lii, G. P., Bloch, C. A., Perna, N. T., Burland, V., Riley, M., ... Shao, Y. (1997). The Complete Genome Sequence of Escherichia coli K-12, 277(September), 1453-1462.

422 chassis, 18, 3403-3424. https://doi.org/10.1111/1462-2920.13230

Bouwer, E. J., \& Zehnder, A. J. B. (1993). Bioremediation of organic compounds - putting microbial metabolism to work. Trends in Biotechnology, 11(August), 360-367.

Chesson, P. (2000). Mechanisms of maintenance of species diversity. Annual Review of Ecology and Systematics, 31, 343-366. https://doi.org/10.1146/annurev.ecolsys.31.1.343

Davison, B. H., \& Stephanopoulos, G. (1986). Effect of pH Oscillations on a Competing Mixed Culture. Biotechnology and Bioengineering, 137, 1127-1137.

Dirk, J., Elsas, V., Chiurazzi, M., Mallon, C. A., Elhottova, D., van Elsas, J. D., ... Salles, J. F. (2012). Microbial diversity determines the invasion of soil by a bacterial pathogen. Proceedings of the National Academy of Sciences of the United States of America, 109(4), 1159-1164. https://doi.org/10.1073/pnas.1109326109 
Ghoul, M., \& Mitri, S. (2016). The Ecology and Evolution of Microbial Competition. Trends in Microbiology, 24(10), 833-845. https://doi.org/10.1016/j.tim.2016.06.011

Göddel, D. V, Kleid, D. G., Bolivar, F., Heyneker, H. L., Yansura, D. G., Crea, R., ... Riggs, A. D. (1979). Expression in Escherichia coli of chemically synthesized genes for human insulin. Proceedings of the National Academy of Sciences of the United States of America, 76(1), 106-110. https://doi.org/10.1073/pnas.76.1.106

Harder, W., \& Veldkamp, H. (1971). Competition of marine psychrophilic bacteria at low temperatures. Antonie van Leeuwenhoek, 37, 51-63.

Höffner, K., \& Barton, P. I. (2014). Design of Microbial Consortia for Industrial Biotechnology. Proceedings of the 8th International Conference on Foundations of Computeraided Process Design (Vol. 34). Elsevier. https://doi.org/10.1016/B978-0-444-63433-7.50008-0

Houde, A., Kademi, A., \& Leblanc, D. (2004). Lipases and their industrial applications: an overview. Applied Biochemistry and Biotechnology, 155-170.

Hsu, B. B., Gibson, T. E., Yeliseyev, V., Bry, L., Silver, P. A., Gerber, G. K., ... Silver, P. A. (2019). Dynamic Modulation of the Gut Microbiota and Metabolome by Bacteriophages in a Mouse Model. Cell Host and Microbe, (25), 803-814. https://doi.org/10.1016/j.chom.2019.05.001

Hutchinson, G. E. (1961). The paradox of the plankton. The American Naturalist, 95(882), 137-145. https://doi.org/10.2307/2678832

Jiang, L., \& Morin, P. J. (2007). Temperature fluctuation facilitates coexistence of competing species in experimental microbial communities. The Journal of Animal Ecology, 76(4), 660-668. https://doi.org/10.1111/j.1365-2656.2007.01252.x 
Koch, B., Jensen, L. E., \& Nybroe, O. (2001). A panel of Tn 7 -based vectors for insertion of the gfp marker gene or for delivery of cloned DNA into Gram-negative bacteria at a neutral chromosomal site.

Litchman, E., Edwards, K. F., \& Klausmeier, C. A. (2015). Microbial resource utilization traits and tradeJournal of Microbiological Methods, 45, 187-195.

Martínez-García, E., Calles, B., Arévalo-Rodríguez, M., \& De Lorenzo, V. (2011). PBAM1: An all-synthetic genetic tool for analysis and construction of complex bacterial phenotypes. BMC Microbiology, 11,

McGrady-Steed, J., Harris, P. M., \& Morin, P. J. (1997). Biodiversity regulates ecosystem predictability. Nature, 390(6656), 162-165. https://doi.org/10.1038/36561

Minty, J. J., Singer, M. E., Scholz, S. A., Bae, C.-H., Ahn, J.-H., Foster, C. E., ... Lin, X. N. (2013). Design and characterization of synthetic fungal-bacterial consortia for direct production of isobutanol from cellulosic biomass. Proceedings of the National Academy of Sciences, 110(36), 14592-14597.

Munna, M. S., Zeba, Z., and Noor, R. (2015). Influence of temperature on the growth of Pseudomonas putida. Stamford Journal of Microbiology, 5(1), 9-12. Diversity predicts stability and resource use efficiency in natural phytoplankton communities. Proceedings of the National Academy of Sciences, 105(13), 5134-5138. 
Rochat, L., Péchy-tarr, M., Baehler, E., Maurhofer, M., \& Keel, C. (2010). Combination of Fluorescent Reporters for Simultaneous Monitoring of Root Colonization and Antifungal Gene Expression by a Biocontrol Pseudomonad on Cereals with Flow Cytometry. Molecular Plant-Microbe Interactions, 23(7), 949-961.

Scholz, S. A., Graves, I., Minty, J. J., \& Lin, X. N. (2017). Production of cellulosic organic acids via synthetic fungal consortia. Biotechnology and Bioengineering, 115(November 2017), 1096-1100. https://doi.org/10.1002/bit.26509

Scott, S. R., Din, M. O., Bittihn, P., Xiong, L., Tsimring, L. S., \& Hasty, J. (2017). A stabilized microbial ecosystem of self-limiting bacteria using synthetic quorum-regulated lysis. Nature Microbiology, 2(June), 1-9. https://doi.org/10.1038/nmicrobiol.2017.83

Shong, J., Rafael, M., Diaz, J., \& Collins, C. H. (n.d.). Towards synthetic microbial consortia for bioprocessing. Current Opinion in Biotechnology, 23(5), 798-802. https://doi.org/10.1016/j.copbio.2012.02.001

Strickland, M. S., Lauber, C. H. L., Fierer, N., \& Bradford, M. A. (2009). Testing the functional significance of microbial community composition. Ecology, 90(2), 441-451.

Thomas, B. S., Koh, O., Bankowski, M. J., \& Seto, T. B. (2013). A lethal case of Pseudomonas putida bacteremia due to soft tissue infection. Infectious Diseases in Clinical Practice, 21(3), 147-213. https://doi.org/10.1097/IPC.0b013e318276956b.A

Thomason, L. C., Costantino, N., \& Court, D. L. (2007). E. coli Genome Manipulation by P1 Transduction. Current Protocols in Molecular Biology, (July), 1.17.1-1.17.8. https://doi.org/10.1002/0471142727.mb0117s79

Tilman, D. (1999). The ecological consequences of changes in biodiversity: A search for general 
principles. Ecology, 80(5), 1455-1474. https://doi.org/10.1890/00129658(1999)080[1455:TECOCI]2.0.CO;2

500

501

502

503

504

505

506

507

508

509

510

511

512

513

Yurtsev, E. A., Conwill, A., \& Gore, J. (2016). Oscillatory dynamics in a bacterial cross-protection mutualism. Proceedings of the National Academy of Sciences (Vol. 113). https://doi.org/10.1073/pnas.1523317113

Zhang, H., Pereira, B., Li, Z., \& Stephanopoulos, G. (2015). Engineering Escherichia coli coculture systems for the production of biochemical products. Proceedings of the National Academy of Sciences, 112(27), 8266-8271. https://doi.org/10.1073/pnas.1506781112

Zhang, S., Merino, N., Okamoto, A., \& Gedalanga, P. (2018). Interkingdom microbial consortia mechanisms to guide biotechnological applications. Microbial Biotechnology, 11, 833-847. https://doi.org/10.1111/1751-7915.13300

Zhou, K., Qiao, K., Edgar, S., \& Stephanopoulos, G. (2015). Distributing a metabolic pathway among a microbial consortium enhances production of natural products. Nature Biotechnology, 33(4), 377383. https://doi.org/10.1038/nbt.3095

Ziv, N., Brandt, N. J., \& Gresham, D. (2013). The Use of Chemostats in Microbial Systems Biology. Journal of Visualized Experiments, (80), 1-10. https://doi.org/10.3791/50168 
Figure 1. Two approaches for rationally regulating synthetic microbial community compositions. A)

517 Under what we refer to as a constant temperature regime, a synthetic bi-culture is kept at a constant

518 temperature throughout the duration of the culture lifetime. The selected temperature determines

519 whether a monoculture persists or co-existence of the two microbes can occur. B) Under a cycling

520 temperature regime a bi-culture is grown in repeated cycles of high and low temperatures, enabling co-

521 existence of the two microbes over an extended period of time. Both the temperatures and the time

522 intervals spent at each temperature can be manipulated to achieve desired outcomes.

524 Figure 2. Maximum specific growth rates of each bacterium in co-cultures with different temperature

525 profiles. E. coli $K 12$ substr. MG1655 grows optimally at relatively higher temperature and Pseudomonas

526 putida KT2440 grows optimally at relatively lower temperatures.

528 Figure 3. Constant temperature regimes can be defined that result in competitive exclusion of either

529 species as well as coexistence. A) Representative flow cytometry data illustrating that when

530 monoculture populations of E. coli-YFP and P. putida-mCh are identified by front scatter/side scatter

531 (grey boxes) $>99 \%$ of E. coli-YFP exhibit fluorescence under our conditions whereas $>99 \%$ of $P$. putida-

$532 m$ Ch do not (blue boxes). B) Representative graphs of species relative abundance over time of bi-culture

533 with varying inoculation ratios grown at different constant temperatures. Each condition has three

534 replicates C) Mean community compositions at each temperature. 
536 Figure 4. The cycling temperature regime is capable of maintaining coexistence of both species in the bi-

537 culture. With alternating temperatures of which one favors E. coli and the other P. putida, each species

538 is spared from competitive exclusion.

540 Figure 5. Effect of time intervals on the amplitude of community composition oscillation. Computational

541 simulation shows that when growth related parameters are kept constant, longer time intervals lead to

542 larger amplitude of oscillation $\left(\mathrm{A}: t^{H}=10.15, t^{L}=15\right)$, whereas shorter time intervals result in more

543 desirable, smaller amplitude of oscillation (B: $\left.t^{H}=3.4, t^{L}=5\right)$. In each graph, the horizontal lines

544 represent the average percentages, which are around 50\%.

546 Figure 6. Experimental results demonstrated that shorter time intervals lead to oscillations of smaller

547 amplitudes, in comparison to those in Fig. 4. 
bioRxiv preprint doi: https://doi.org/10.1101/2020.02.14.944090; this version posted February 14, 2020. The copyright holder for this preprint Ahich was not cartified by peer review) is the author/funder whohas granted bioRxiv a license to display the preprint in perpetuity. It is made
.
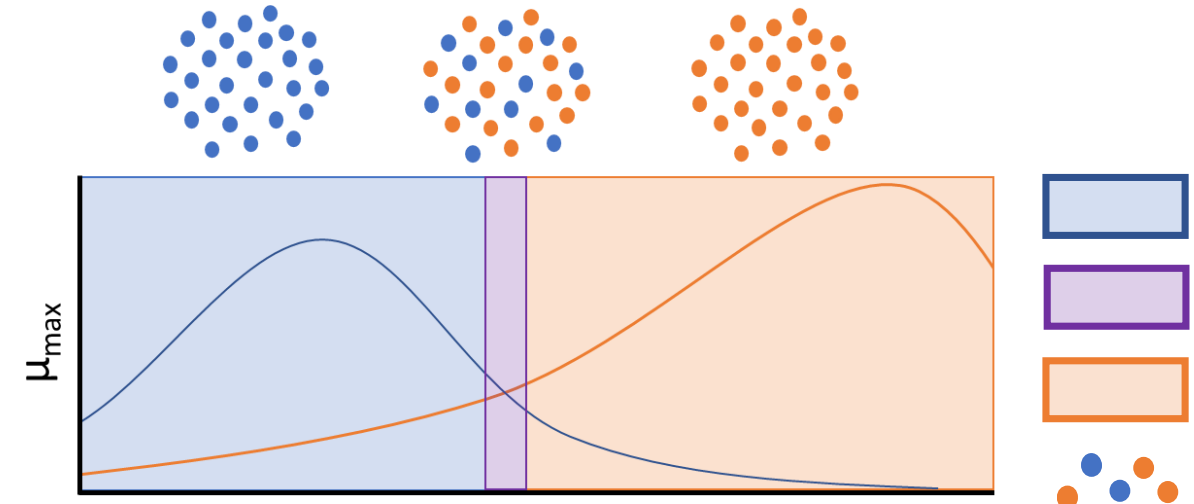

Species A favored temperature range

Coexistence temperature range

Species B favored temperature range

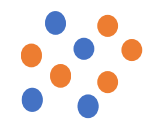

Community composition

Temperature

B. Cycling temperature regime

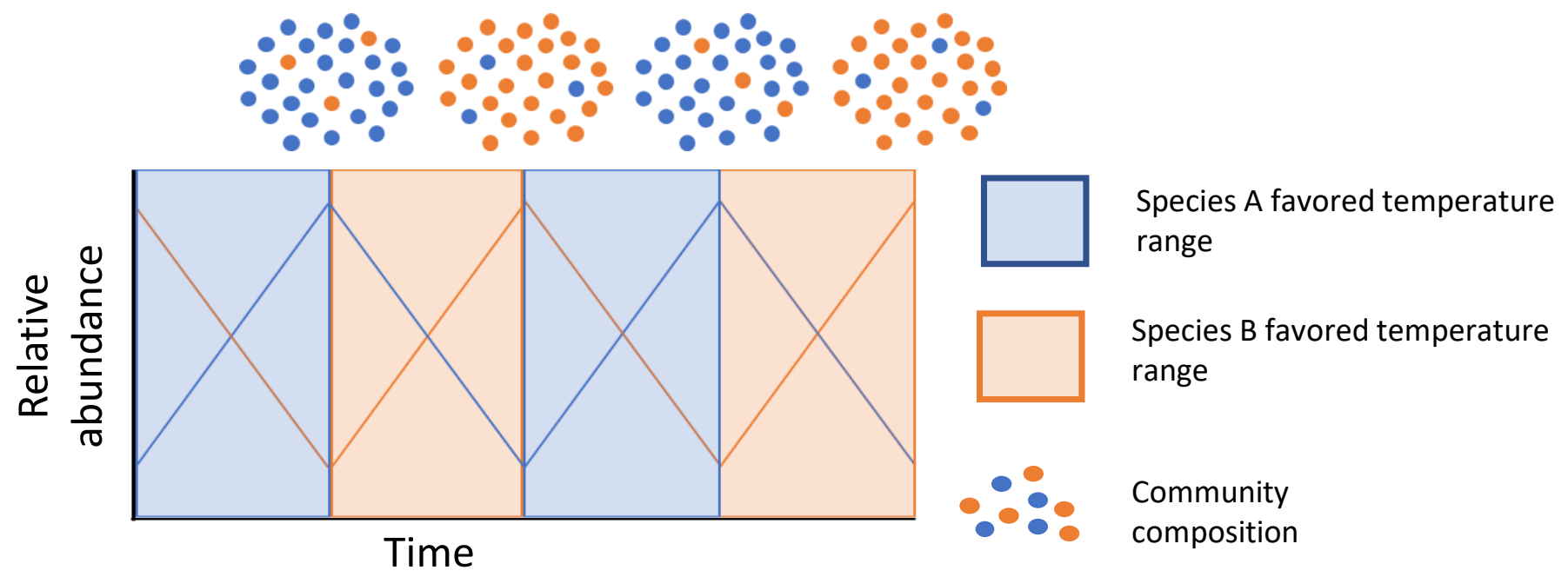


bioRxiv preprint doi: https://doi.org/10.1101/2020.02 14.944090; this version posted February 14,2020 . The copyright holder for this preprint (which was not certified by peer review) is the author/funder, who has granted bioRxiv a license to display the preprint in perpetuity. It is made available under aCC-BY 4.0 International license.

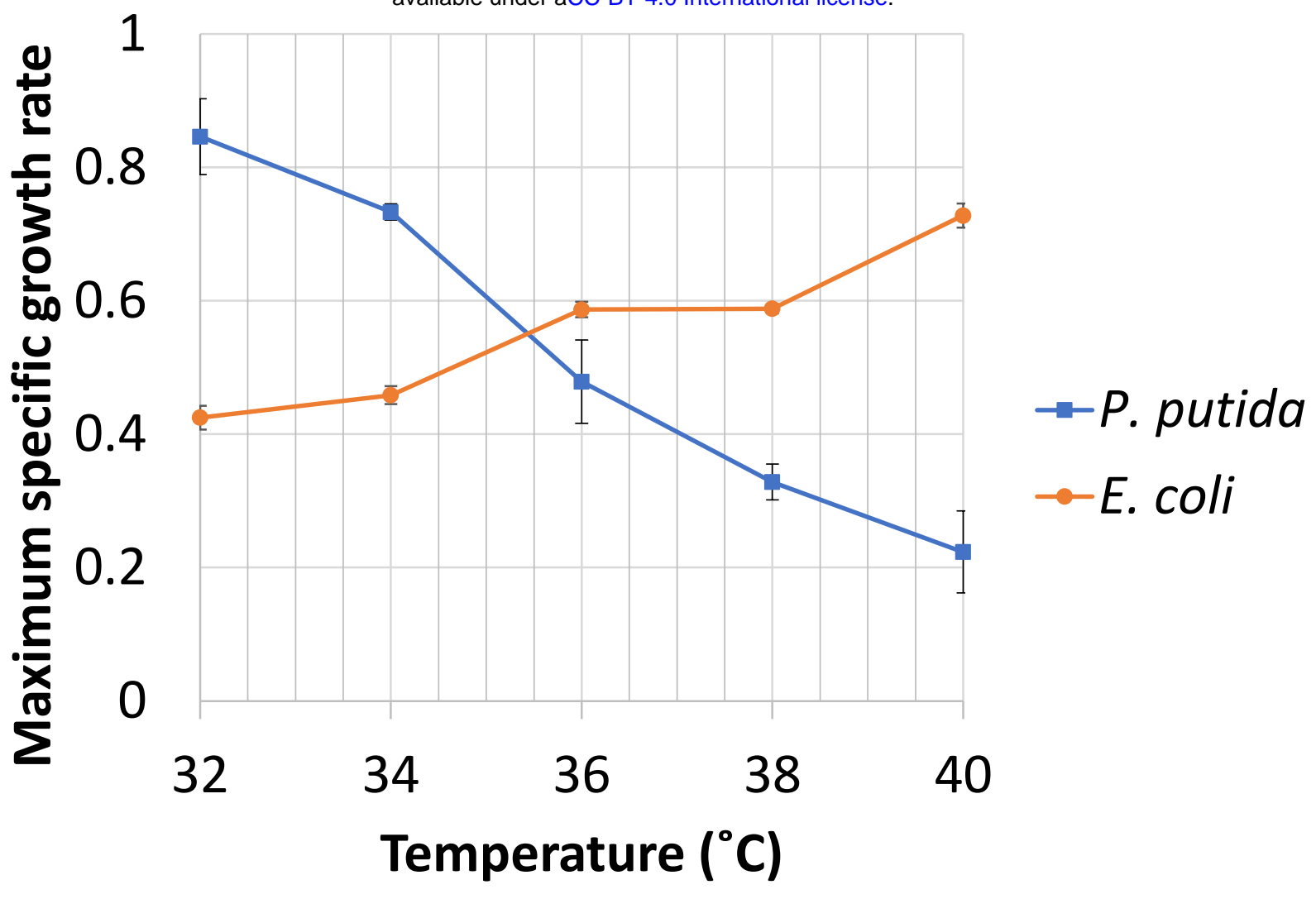


bioRxiv preprint doi: https://doi.org/10.1101/2020.02 14.944090; this version posted February 14,2020. The copyright holder for this preprint (which was not certified by peer review) is the author/funder, who has granted bioRxiv a license to display the preprint in perpetuity. It is made

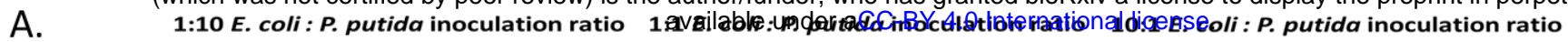

$35.5^{\circ} \mathrm{C}$

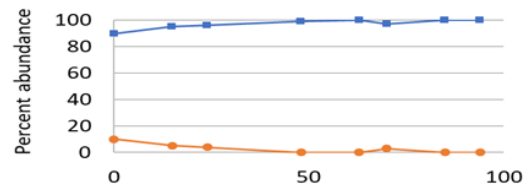

$36.5^{\circ} \mathrm{C}$

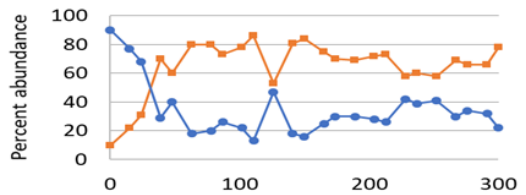

$39.5^{\circ} \mathrm{C}$

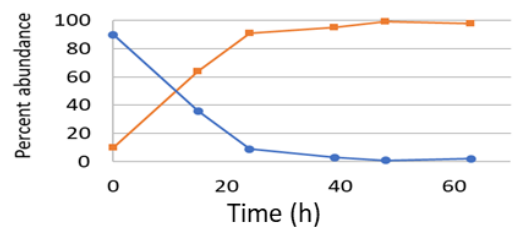

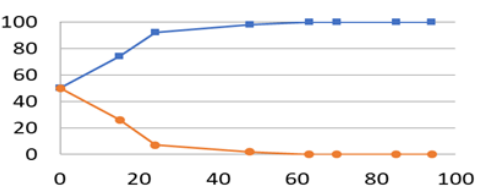
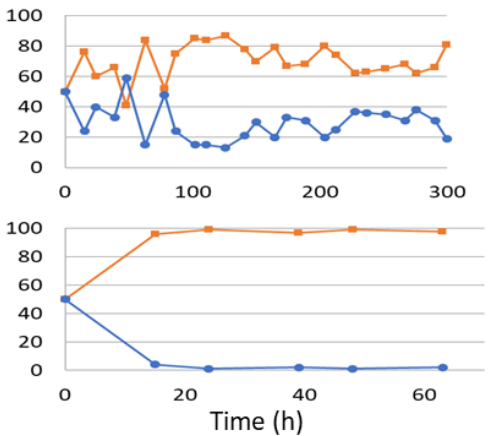
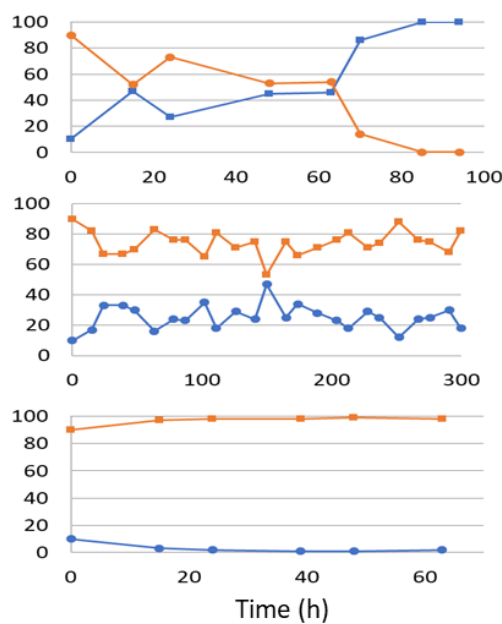

B.

Mean community composition across temperatures

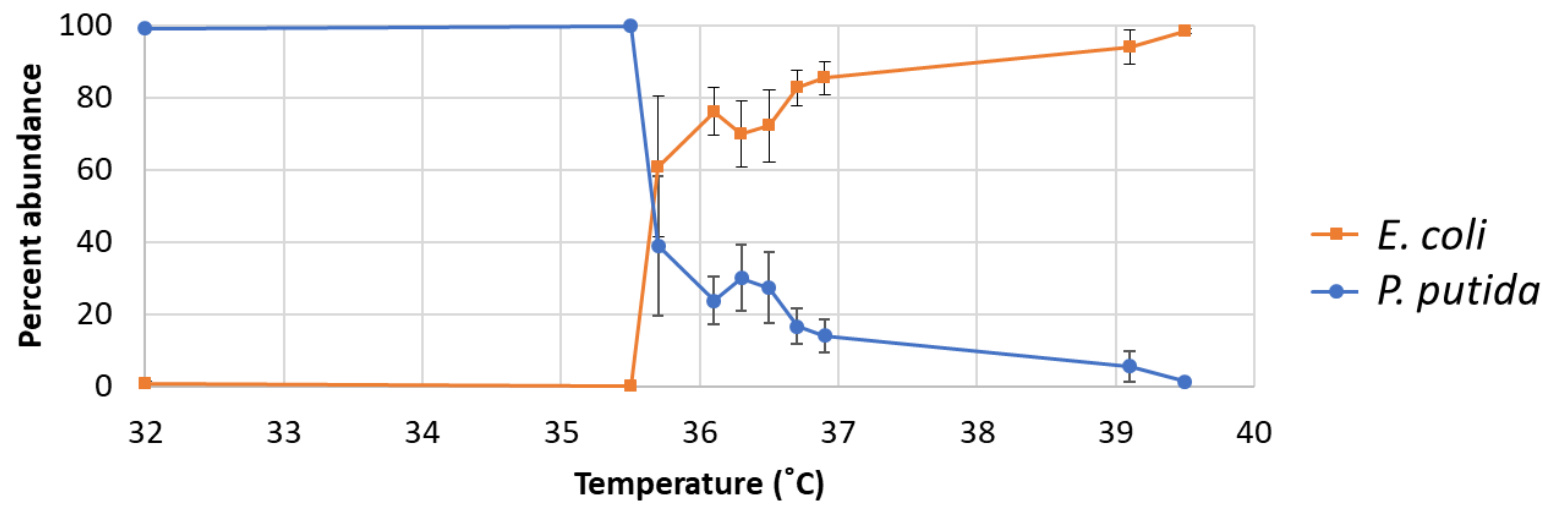


bioRxiv preprint doi: https://doi.org/10.1101/2020.02 14.944090; this version posted February 14,2020 . The copyright holder for this preprint (which was not certified by peer review) is the author/funder, who has granted bioRxiv a license to display the preprint in perpetuity. It is made available under aCC-BY 4.0 International license.

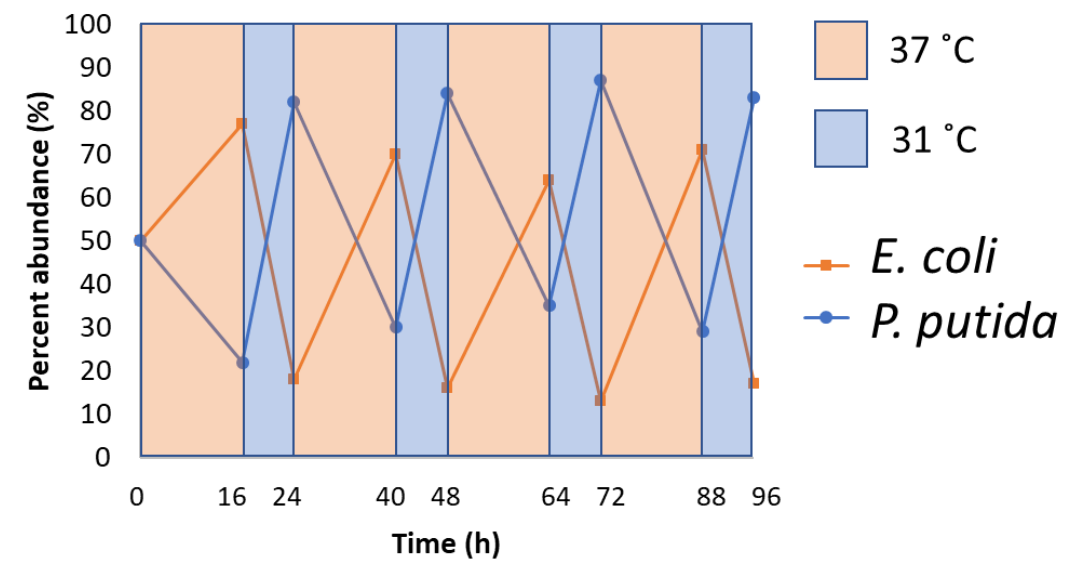




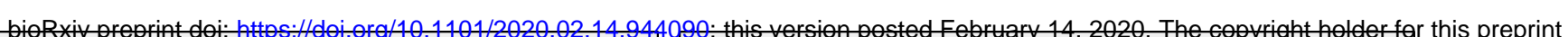
(which was not certified by peer review) is the author/funder, who has granted bioRxiv a license to display the preprint in perpetuity. It is made
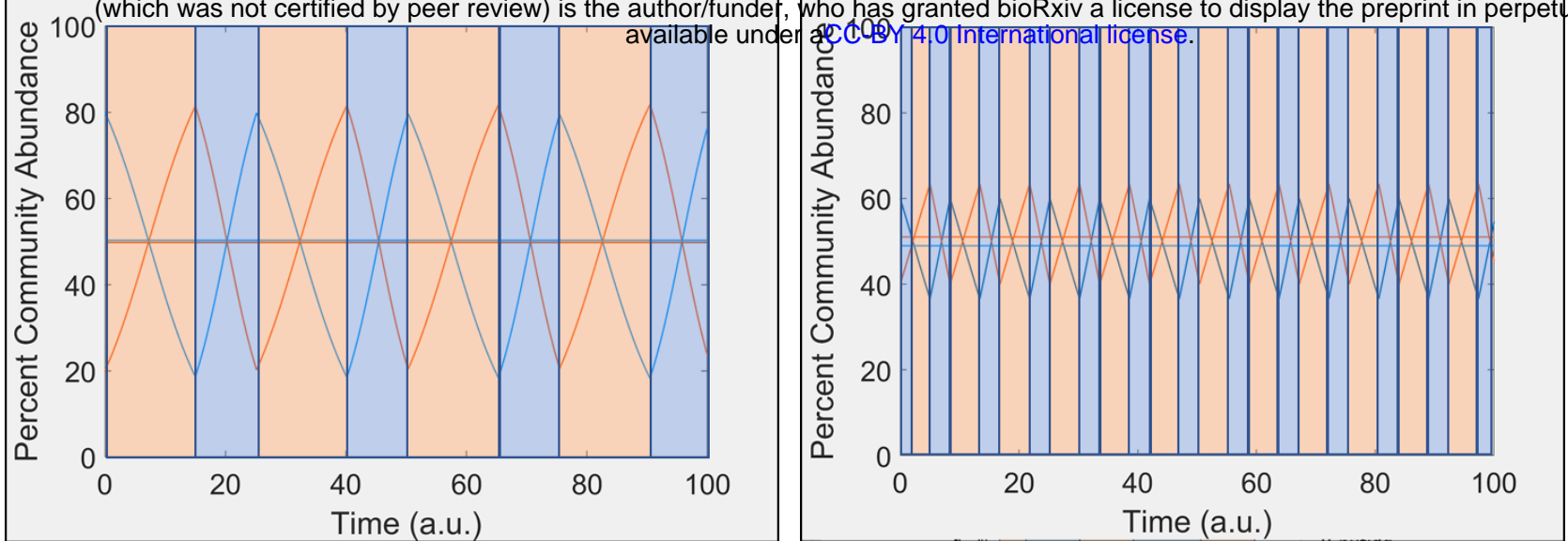

- E. coli

$\rightarrow$ P. putida 
bioRxiv preprint doi: https://doi.org/10.1101/2020.02.14.944090; this version posted February 14, 2020. The copyright holder for this preprint (which was not certified by peer review) is the author/funder, who has granted bioRxiv a license to display the preprint in perpetuity. It is made available under aCC-BY 4.0 International license.

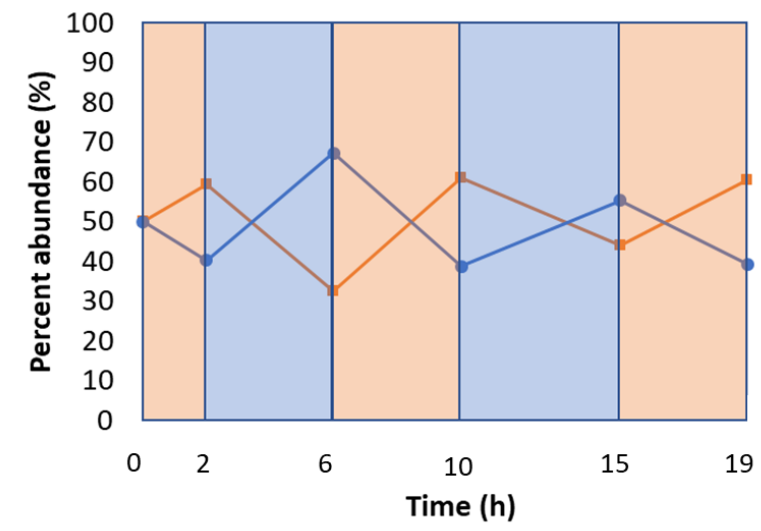

$\square 39{ }^{\circ} \mathrm{C}$

$27^{\circ} \mathrm{C}$

E. coli

$\rightarrow$ P. putida 